\title{
A New Formula of Redshift vs. Space Expansion and Dark Energy
}

\author{
Olivier Serret \\ ESIM Engineer, Cugnaux, France \\ Email: o.serret@free.fr
}

How to cite this paper: Serret, O. (2021) A New Formula of Redshift vs. Space Expansion and Dark Energy. Journal of Modern Physics, 12, 229-253.

https://doi.org/10.4236/jmp.2021.123018

Received: December 23, 2020

Accepted: February 17, 2021

Published: February 20, 2021

Copyright $\odot 2021$ by author(s) and Scientific Research Publishing Inc. This work is licensed under the Creative Commons Attribution International License (CC BY 4.0).

http://creativecommons.org/licenses/by/4.0/

\begin{abstract}
The speed away of stars and galaxies is traditionally calculated from the relativistic formula according to the measurement of the redshift. It is demonstrated here another formula for this speed away of stars and galaxies, from this same redshift $z$. After having exposed critiques on the demonstration and the relativistic use which require the assumption of an expanding universe by itself, it is proposed within the framework of neo-Newtonian mechanics the formula $V=\frac{z}{1+z} c$ where $V$ is the speed from the source. This formula has the double characteristic of being equivalent to relativistic predictions for low redshifts, but of deviating from them by up to $17 \%$ for high redshifts. It is consistent with the observation of the anisotropy of the Universe and the Cosmic Microwave Background. It helps to explain Pioneer anomalies. It is compatible with the constancy in the majority of cases of interference phenomena. Finally, it provides a new analysis grid for the observation of supernovae, galaxies and distant pulsars, thus opening up new fields of research on the increase in distances attributed to dark energy and also in other areas of the cosmology.
\end{abstract}

\section{Keywords}

Redshift, Blueshift, Dark Energy, Radial Velocity, Expansion of the Universe, Anisotropy, Hubble Law, Pioneer Spacecraft, Photon, Wavelength, Frequency, Variable Speed, Celerity of Light

\section{Introduction}

In the past, the stars did not exactly follow the supposedly perfectly circular trajectory, it was necessary to imagine the existence of epicycles ... until new elliptical trajectories were accepted. Today, the distant galaxies not having to exceed 
the speed of light in spite of the law of Hubble, it is imagined a space which would create itself its own space, and the distant supernovae not following exactly the speed away calculated there, it is imagined the existence of a repulsive dark energy ... unless we come to accept a new formula for the speeds away as a function of the redshift. This is what we will try to demonstrate in this article.

After having recalled the definitions and uses of redshift and wavelength, we will analyze the research status on the relativistic redshift formula. Then we will propose the neo-Newtonian derivation to get to the expression of a slightly modified formula. Finally, we will discuss the consequences or not of this change of formula.

\section{Definition and Context}

The redshift, noted $z$, is the fringe shift measured on light and electromagnetic waves from moving objects. From this measurement, first the speed away for stars and galaxies is calculated and then their distance is deduced using Hubble's "law".

Yet, at the horizons of the Universe, by correlating with the distances derived from their luminosity, supernovae appear to be at distances greater than those calculated by the redshift method (see Figure 1). To explain this additional distance speed and therefore this additional distance, it is invoked the existence of a dark energy with a repulsive effect, dark energy which has so far not been able to be directly apprehended. And more simply, why not question the formulas used to calculate the very fast speeds and the far distances?

Light and electromagnetic waves in general are characterized by their wavelength $\lambda$, their propagation speed $c$ and their frequency $f$.

We can characterize them only by two out of three parameters because they are related to each other by the relation:

$$
\lambda=c / f
$$

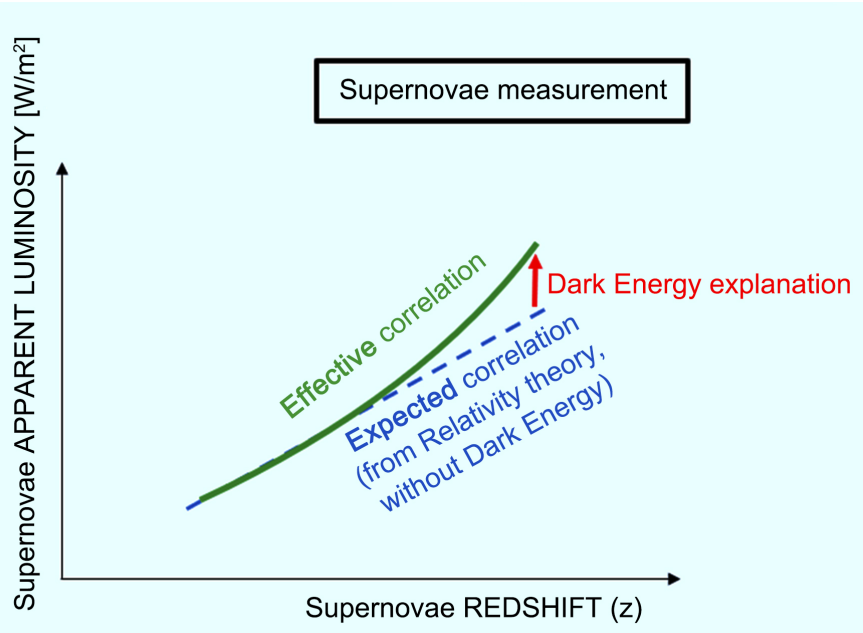

Figure 1. Schematic explanation of Dark Energy. 
Since the speed of light in vacuum is assumed to be constant, it has become customary to characterize the electromagnetic waves by only one parameter, the wavelength $\lambda$ (i.e. a distance). Yet this is the frequency (i.e. a time, or more exactly the inverse of time) that we perceive or directly measure. For example, an optical detector does not measure a length, but is excited at certain frequencies. This is why in this article we will favor the explanation by frequencies $f$ (rather than by wavelengths $\lambda$ ).

\section{Analysis of the Relativistic Redshift Formula}

The relativistic expression allowing to deduce the distance speed $V$ from the redshift $z$ is as follows:

$$
V=-\frac{(1+z)^{2}-1}{(1+z)^{2}+1} c
$$

This formula comes up against two difficulties.

\subsection{The Derivation Itself}

The usual demonstration goes through the following intermediate step [1] [2] [3] [4]:

$$
\lambda_{o b s}=\frac{c-V}{f_{o b s}}
$$

The problem is that the theory of Relativity postulates that the speed linking the two quantities $\lambda_{o b s}$ and $f_{o b s}$ is constant and therefore that the numerator must be equal to $c$ in any frame of reference. This means that $V$ should be zero, which is contradictory with a moving source.

There are also few much more complex derivations, but the one we have seen [5] is also open to criticism (in this video, there is a mix between Galilean formulas and relativistic formulas and the Equation (3) is implicit).

\subsection{The Expansion of Space Itself}

Even admitting the validity of the previous derivation, the relativistic formula only allows to relate the redshift to the speed by making an additional assumption, namely the expansion of space itself. This additional assumption does not appear necessary as long as the source does not move away at very high speeds because it is used a simplified formula. But when they are faced with very high speed or high $z$ s, they end up with an inconsistency that can only be overcome by posing the strange hypothesis of a space that expands by itself. Without this additional assumption, the relativistic Equation (2) of the redshift leads to an inconsistent situation on the propagation speeds [see Appendix 1].

\section{The Neo-Newtonian Derivation}

\subsection{The Neo-Newtonian Mechanic}

So what formula should be used between the distance velocities and the fringe 
shifts received from high- $Z$ stars and galaxies? The proposal falls within the framework of neo-Newtonian mechanics which is none other than Newtonian mechanics but without the principle of equivalence between inert mass and gravitational mass: thus, the increase of inertial mass as a function of the speed prevents the photon from exceeding the celerity $c$, this means that the speed of the photon would not be constant in any frame of reference. The purpose of this article is not to detail Newtonian Mechanics, the reader may consult it in the RELATED LINKS [a]. And as in Newtonian mechanics, it is assumed that the photon is a body of matter that can move in a vacuum, and that there is a fixed or barycentric frame $R_{0}$.

\subsection{The Method of the Derivation}

The derivation is based on the redshift understood as an effect of the Doppler-Fizeau effect. The Doppler-Fizeau effect can be described as the effect produced by a moving source of electromagnetic waves in which there is an apparent upward shift in frequency for observers towards whom the source is approaching and an apparent downward shift in frequency for observers from whom the source is receding. Here is a simplified derivation of the speed away as a function of redshift, the general derivation is given in Appendix 2. Let us take the usual and simplified case of a photon emitted from a source which moves away while the observer is motionless in the fixed frame of reference $R_{0}$. The photons (A) then (B) are emitted according to the time interval $T_{\text {source }}$ at the celerity $c$ in the reference frame of the source, but according to neo-Newtonian mechanics they move at the speed $(c-V)$ in the fixed reference frame $R_{0}$ (see Figure 2)

$$
v_{\text {photon } / R_{0}}=c_{\text {photon } / \text { source }}-\left|v_{\text {source } / R_{0}}\right|
$$

with $\left|v_{\text {source/ } R_{0}}\right|>0$.

There are two ways to calculate the distance between photons (A) and (B):

- The first way is to note that the distance $\lambda$ between the photons (A) and (B) is the distance $D_{A}$ traveled by the photon (A) during the time $T_{\text {source }}$ added to the distance $d_{\text {source }}$ traveled by the source until the emission photon (B) (see Figure 3)

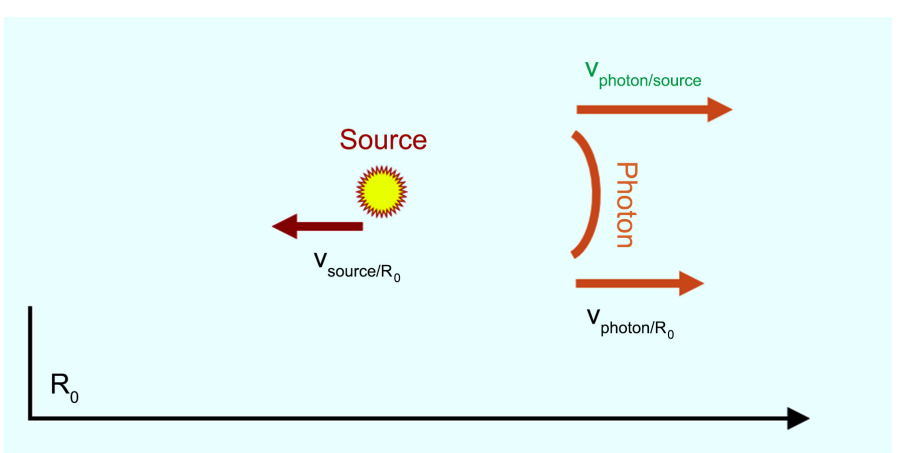

Figure 2. Velocities of the photon: in black/Ro and in green/source S. 


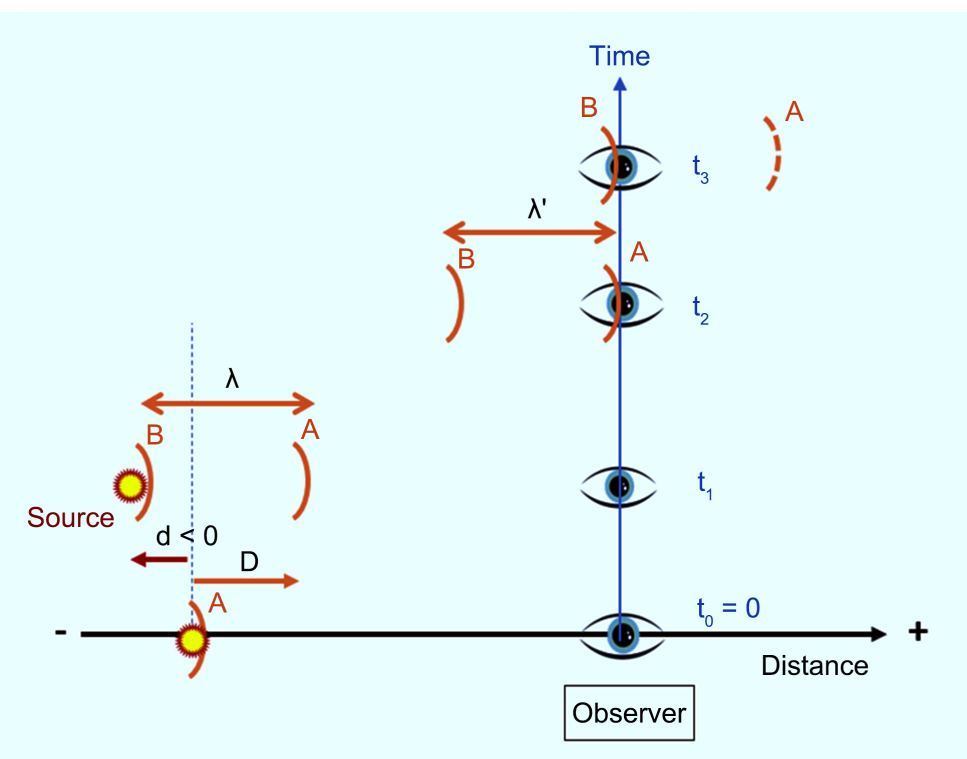

Figure 3. Photons from the moving source to the observer.

$$
\begin{gathered}
\lambda=D_{A}+\left|d_{\text {source }}\right| \\
\lambda=v_{A / R_{0}} * T_{\text {source }}+\left|v_{\text {source } / R_{0}} * T_{\text {source }}\right|
\end{gathered}
$$

- The second way is to consider that the distance between the photons is the propagation distance of the photon (B) until it reaches the position where the photon (A) had been observed at the time of the emission of (B):

$$
\begin{aligned}
& \lambda^{\prime}=v_{B / R_{0}} * T_{o b s} \\
& \lambda^{\prime}=v_{B / R_{0}} / f_{o b s}
\end{aligned}
$$

In neo-Newtonian mechanics, there is no variation in distance or time depending on the speed of the frame of reference, the distance between two photons is the same whether it is calculated from the observer or from the source.

$$
\lambda^{\prime}=\lambda
$$

The frequency being the inverse of the period, the redshift is defined from the frequencies by:

$$
1+z=\frac{f_{\text {source }}}{f_{\text {obs }}}
$$

we then get (see details of the calculation in Appendix 2):

$$
v_{\text {source }}=-\frac{z}{1+z} c
$$

And in the case where the speed of the source with respect to the frame of reference $R_{0}$ is directed in the same direction as the photon, the formula is then

$$
v_{\text {source }}=z \cdot c
$$

In both cases, it is a Doppler-Fizeau effect applied to electromagnetic waves, without expansion of the space itself. 


\section{Discussion}

\subsection{The Difference with the Relativistic Speed Prediction}

Compared to the relativistic redshift formula, the new speed away remains lower than the previous one until it reaches $17 \%$ deviation at $z=1.4$ as shown in Figure 4. This is not incompatible with the current estimate of $+/-10 \%$ of distance [6]. And that means that the neo-Newtonian formula is closer to relativistic predictions than to Newtonian predictions. However, note that for usual values up to $Z=0.02$ (i.e. according to Hubble's law up to 240 million light years), the difference does not exceed 1\% (See Table 1 ). And for slightly smaller values up to $Z$ $=0.01$ we find within $1 \%$ the usual approximation

$$
v_{\text {source }}=-z \cdot c
$$

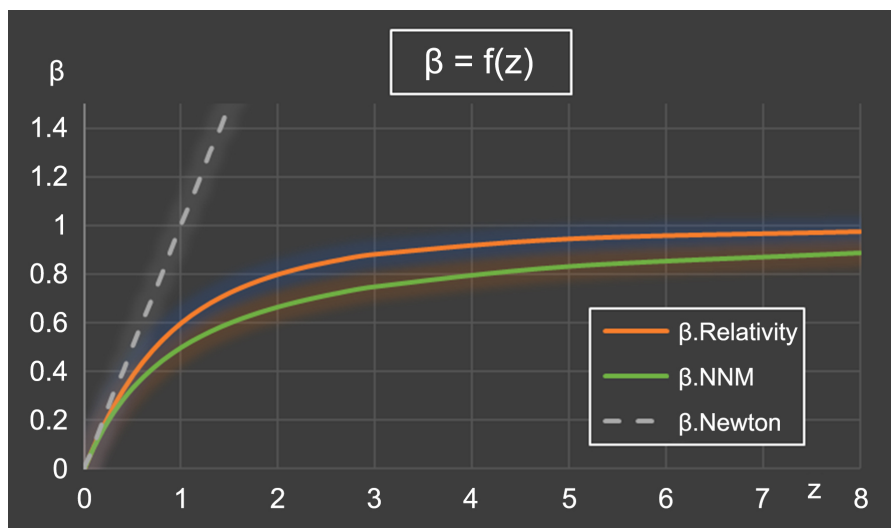

Figure 4. Velocities in Relativity theory, Newtonian mechanics and neo-Newtonian mechanics.

Table 1. Comparison of velocities.

\begin{tabular}{cccccc}
\hline VELOCITY & $\beta=\mathbf{z}$ & Relativity & Neo Newtonian & \multicolumn{2}{c}{ Difference } \\
\hline $\mathbf{z}$ & {$[\mathrm{km} / \mathrm{s}]$} & {$[\mathrm{km} / \mathrm{s}]$} & {$[\mathrm{km} / \mathrm{s}]$} & {$[\mathrm{km} / \mathrm{s}]$} & {$[\%]$} \\
\hline 0 & 0 & 0 & 0 & 0.0 & $0.0 \%$ \\
0.001 & 300 & 300 & 300 & 0.1 & $0.0 \%$ \\
0.005 & 1500 & 1496 & 1493 & 4 & $0.2 \%$ \\
0.01 & 3000 & 2985 & 2970 & 15 & $0.5 \%$ \\
0.02 & 6000 & 5940 & 5882 & 58 & $1 \%$ \\
0.1 & 30,000 & 28,507 & 27,273 & 1234 & $4 \%$ \\
0.5 & 150,000 & 115,385 & 100,000 & 15,385 & $13 \%$ \\
1 & 300,000 & 180,000 & 150,000 & 30,000 & $17 \%$ \\
1.6 & 480,000 & 222,680 & 184,615 & 38,065 & $17 \%$ \\
2 & 600,000 & 240,000 & 200,000 & 40,000 & $17 \%$ \\
5 & $1,500,000$ & 283,784 & 250,000 & 33,784 & $12 \%$ \\
8 & $2,400,000$ & 292,683 & 266,667 & 26,016 & $9 \%$ \\
15 & $4,500,000$ & 297,665 & 281,250 & 16,415 & $6 \%$ \\
1000 & $300,000,000$ & 299,999 & 299,700 & 299 & $0 \%$ \\
\hline
\end{tabular}


Thus, the difference in prediction with the relativistic values of speed only appears significant for very large redshift values $z$ exceeding unity.

\subsection{A Universe Which Does Not Expand Itself}

In the relativistic demonstration of redshift it is implied a space which expands itself. This assumption is also found with the law of Hubble which would like that the galaxies located on the cosmic horizon, to 13.8 billion light years, would go in the future faster than the speed of the light except to consider also that space would expand on its own. And this space would be expanding between galaxies but not within galaxies, as evidenced by the image of raisin bread in an oven [7]. This strange hypothesis is akin to "creationism". The derivation of the neo-Newtonian formula does not come up against these difficulties. With a source moving away from a fixed observer, we calculate the distance $d$ traveled by the source, and we calculate the distance $L$ that the photon (B) travels. When we make the difference $\delta$ of the distances, it is zero. And for blueshift with a source that approaches a fixed observer, the difference $\delta$ in distances is also zero. In these two cases, with neo-Newtonian mechanics there is no longer any need to consider an expanding space (see Appendix 3).

\subsection{Interference of Light Waves}

Having a variable frequency and a variable propagation speed makes it possible to have an unchanged wavelength. This is why this generally does not change the light interference phenomena and why it is difficult to see it by this means (see Appendix 4).

\subsection{The Existence of a Fixed Frame of Reference}

The hypothesis of a fixed or barycentric frame of reference $R_{0}$ is consistent with the latest observations on the anisotropy of the Universe [8] [9] [10] (see Figure 5) who measured that the solar system is moving at $390 \mathrm{~km} / \mathrm{s}$ compared to the Diffuse Cosmological Background, the Galaxy having to move at $630 \mathrm{~km} / \mathrm{s}$ (more than two million $\mathrm{km} / \mathrm{h}$ ). This Cosmic Microwave Background (CMB) would thus play this role of reference frame $R_{0}$.

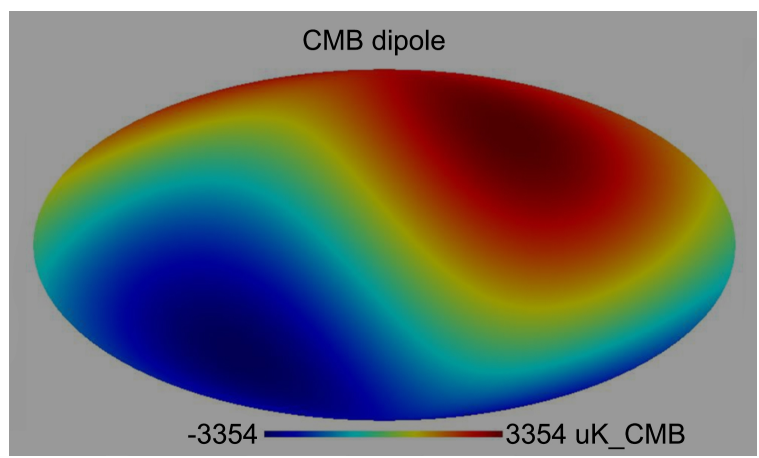

Figure 5. Dipolar anisotropy of the CMB (COBE measurement) before treatment [11]. Color bar: red for redshift, blue for blueshift. 


\subsection{Proof by the Pioneer Spacecrafts}

The Pioneer spacecrafts had two characteristics, their very high speed at $37 \mathrm{~km} / \mathrm{s}$ after the passage of Jupiter and the absence of stabilization or correction of the flight (which the Voyager spacecraft are equipped with). Examination of the trajectory of the two Pioneer spacecraft had detected complementary retrograde acceleration - that is, an unexplained slowing down. Officially this anomaly was solved by decreeing that this acceleration came from a continuous jet of photons emitted by each spacecraft. Nevertheless, in a previous article [e], we had shown that this anomaly could also be easily explained by taking the empirical relation:

$$
1+z=\frac{1}{1-|\beta|}
$$

$\beta$ was then oriented in the other direction, but this formula is strictly equivalent to that demonstrated in this article. So this new formula for redshift helps explain the Pioneer anomaly or rather conversely, Pioneer spacecrafts demonstrate the validity of this formula for redshift.

\subsection{A New Paradigm}

Having a lower distance velocity does not necessarily mean that the distances would be lower. Indeed, relativistic cosmology is based on Hubble's law, with a Hubble constant which is paradoxically not constant over time and whose value differs depending on the measurement method used. The greater than expected distances and speeds prevalent by dark energy proponents could just as easily mean a current slowing of the expansion due to gravity. Recall that a traditional explosion also implies that all bodies move away from each other in proportion to their respective distance. No longer correlating the speed of the redshift with Hubble's law would make it possible to have another interpretation of distance, for example for quasars whose apparent luminosity is poorly explained [12]. This would allow us to re-examine the age of the Universe by taking into account the age of stars estimated to be over 14 billion years old [13]. The different distances (luminous, covariant, angular, proper and cosmological), all linked by the redshift $z$, could surely be simplified by considering that basically there are in relation to the observer only two distances, that of emission and that where the body is now. These are just examples of possible developments. Having a new paradigm opens up the fields of the possible with new areas to test, study and interpret

\section{Conclusions}

We have derivated within the framework of neo-Newtonian mechanics the value $V$ of the speed from the source in function of the redshift to get to a new formula: $V=\frac{z}{1+z} c$. This formula has the double characteristic of being equivalent to relativistic predictions for low redshifts, but of deviating from them by up to $17 \%$ for high redshifts. 
What can be the consequences of future research?

For any observed physical phenomenon, we seek an explanation. For example:

1) As the speed of expansion of the Universe cannot exceed the speed of light, it is assumed that space itself is expanding.

2) As the light interference remains identical despite the movement of the source, it is assumed that the speed of light would be constant in any frame of reference.

3) As our Galaxy being in motion with respect to the Cosmic Microwave Background (in contradiction with the isotropy of the theory of Relativity), it is assumed that it would be subjected to the Dipole Repeller.

4) As the Pioneer spacecrafts seem to be slowing down more than expected, it is assumed that they would be slowed down by their own flow of photons.

5) As distant supernovae appear less bright than expected, it is assumed that there is a dark energy that makes them accelerate.

Faced with these difficulties of interpretation, we propose here a change of paradigm, namely that resulting from neo-Newtonian mechanics:

1) We have expressed and demonstrated a slightly different formula relating the redshift to the speed of movement of the source. Thus, it is no longer necessary to imagine an expanding space by itself, which does not prevent galaxies from moving away from each other as a result of an explosive-type phenomenon.

2) We have shown that a variable frequency and speed of propagation depending on the movement of the source can explain a constant wavelength and therefore stable light interferences.

3) The observed anisotropy of the Universe can be explained very well by considering the Cosmic Microwave Background as the expression of a fixed or barycentric frame $R_{0}$.

4) The slight blueshift effect of Pioneer spacecrafts is proof of the validity of this new redshift formula:

5) The Universe retains many mysteries, and not just about distant supernovae and quasars.

This new analysis grid proposed here (within Neo-Newtonian Mechanics) would allow any researcher interested in it to shed new light on his research, to test it and would surely open up the field of possibilities.

\section{Acknowledgements}

I would like to thank BGD for the reading and for the support.

\section{Conflicts of Interest}

The author declares no conflicts of interest regarding the publication of this paper.

\section{References}

[1] Vincent, M. (2012) Validation des principes de la relativité restreinte (3/3). ENS 
Lyon.

http://culturesciencesphysique.ens-lyon.fr/ressource/validation-relativite-restreinte3.xml

[2] Chodorowski, M.J. (2011) Monthly Notices of the Royal Astronomical Society, 413, 585-594. https://arxiv.org/abs/0911.3536 https://doi.org/10.1111/j.1365-2966.2010.18154.x

[3] Langlois, D. (2011) Introduction à la relativité, cours et exercices.Vuibert, p. 30.

[4] Semay, C. and Silvestre-Brac, B. (2016) Relativité restreinte, bases et applications. Dunod, p. 179.

[5] Guth, A. (2013) 3. The Doppler Effect and Special Relativity. MIT. https://www.youtube.com/watch?v=tJ2AJJMcQXs

[6] Luri, X., et al. (2018) $A \& A, 616,2-19$. https://www.aanda.org/articles/aa/pdf/2018/08/aa32964-18.pdf

[7] Courtois, H. (2020) Voyage sur les flots de galaxies, Laniakea et au-delà. Dunod.

[8] Rubin, V.C. (1977) Is There Evidence of Anisotropy in the Expansion of the Universe? Cambridge University Press, Cambridge. https://doi.org/10.1017/S0252921100053549

[9] Colin, J., et al. (2019) A\&A, 3-7. https://arxiv.org/pdf/1808.04597.pdf

[10] Migkas, K., et al. (2020) $A \& A, 636$, p. 42. https://doi.org/10.1051/0004-6361/201936602

[11] NASA, LAMDA-DMR Images. https://lambda.gsfc.nasa.gov/product/cobe/dmr_image.cfm

[12] Arp, H. (1967) Astrophysical Journal, 148, p. 321. https://ui.adsabs.harvard.edu/abs/1967ApJ...148..321A/abstract https://doi.org/10.1086/149159

[13] Bond, H.E., et al. (2013) The Astrophysical Journal Letters, 765, 1-2, 4. https://doi.org/10.1088/2041-8205/765/1/L12 


\section{Related Links}

Let's free Newtonian Mechanics from the equivalence "principle"!

a Presentation https://www.gsjournal.net/Science-Journals/Research\%20Papers-Relativity\%20Th eory/Download/7499

How to Demonstrate the Lorentz Factor. Variable Time vs. Variable Inertial Mass http://file.scirp.org/pdf/JMP_2015022510573131.pdf

Lorentz derivation Reply to "A Simple Derivation of the Lorentz Transformation" http://file.scirp.org/pdf/JMP_2017121915203275.pdf

Lorentz transformation derivation criticism https://www.youtube.com/watch?v=koPnW0mXcvI

Net Force $F=\gamma^{3}$ ma at High Velocity http://file.scirp.org/pdf/JMP_2016042814580505.pdf

Velocity Addition Demonstrated from the Conservation of Linear Momenta, an Alternative Expression http://file.scirp.org/pdf/JMP_2015050609513342.pdf Mass of Inertia and Kinetic Energy http://gsjournal.net/Science-Journals/Research\%20PapersRelativity\%20Theory/D ownload $/ 4113$

An improvement of the accuracy of Fizeau's experiment

http://gsjournal.net/Science-Journals/Research\%20Papers-Relativity\%20Theory/ Download/7247

The new speed composition $u=v+w / \gamma^{2}$ in agreement with Fizeau's experiment http://gsjournal.net/Science-Journals/Research\%20Papers-Relativity\%20Theory/

Download/7247

$X X X X$

Present one

e Redshift

The Pioneer Anomaly explained by the Processing of the Doppler Effect http://gsjournal.net/Science-Journals/Research\%20Papers-Relativity\%20Theory/ Download/7330

Redshift derivation criticism

f Michelson experiment

g Sagnac effect

$\mathrm{h}$

Muons

Lifetime

Mercury

perihelion

Which derivation for the result of the MMX (Michelson \& Morley Experiment) in translation with respect to the observer?

https://www.gsjournal.net/Science-Journals/Research\%20Papers-Relativity\%20Th eory/Download/7808

A Non-Relativistic Explanation of the Sagnac Effect https://file.scirp.org/pdf/JMP_2019041715043977.pdf

Muon Lifetime would depend of its Energy

http://www.mrelativity.net/Papers/51/Muons\%20Serret\%20Millennium.pdf

About the ovoid orbits in general, and perihelion precession of Mercury in particular (2)

http://www.mrelativity.net/Papers/51/Mercury\%20Millennium\%20Serret\%205\%2 0janvier\%202018.pdf

Hipparcos did not measure directly the light bending! http://gsjournal.net/Science-Journals/Research\%20Papers-Mechanics\%20/\%20Ele

Light

deflection ctrodynamics/Download/6998

Gravitational Light Deflection: could Relativity be Invalidated by GAIA? http://mrelativity.net/Papers/51/Light\%20deflection\%20SERRET\%20Millennium \%20Aout\%202018.pdf 


\section{Continued}

Shapiro time Shapo Time Delay derivates from Refraction \%20SERRET\%20Millennium\%20jui llet\%202018.pdf Matter https://physicsessays.org/browse-journal-2/product/1240-7-olivier-serret-the-flatrotation-curve-of-our-galaxy-explained-within-newtonian-mechanics.html

m Dark Energy
Gravity vs. Dark Energy, about the Expansion of the Universe http://file.scirp.org/pdf/JMP_2018011714405269.pdf

Gravitational waves or particle radiation?

n Gravitational https://www.physicsessays.org/browse-journal-2/product/1588-12-olivier-serret-g waves ravitational-waves-or-particle-radiation.html 


\section{Appendix 1: Criticism of the Derivation of the Relativistic Redshift Formula}

Let us have

- An observer without motion (he is in his own frame)

- A source moving at the velocity $v_{\text {source }}$ (in the observer's frame). So

$$
\beta=\frac{v_{\text {source }}}{c}
$$

Relativistic equation hereafter

$$
1+z=\sqrt{\frac{1+\beta}{1-\beta}}
$$

is equivalent to

$$
\beta=\frac{(1+z)^{2}-1}{(1+z)^{2}+1}
$$

The links between the frequencies and the periods are

$$
\begin{aligned}
& 1+z=\frac{f_{\text {source }}}{f_{\text {obs }}} \\
& 1+z=\frac{T_{\text {obs }}}{T_{\text {source }}}
\end{aligned}
$$

We'll check two cases, the redshift and the blueshift one.

1) Edshift Case

See specific configuration of Figure 6.

- The distance of emission source of the photon (B) at the time $t_{1}=T_{\text {source }}$ :

$$
\begin{gathered}
d_{\text {source }}=v_{\text {source }}\left(t_{1}-t_{0}\right) \\
d_{\text {source }}=-\beta \cdot c \cdot T_{\text {source }}
\end{gathered}
$$

with $v_{\text {source }}$ and so $\beta<0$.

- And the length traveled by the photon (B) to reach the observer is

$$
\begin{aligned}
L & =v_{\text {photon }}\left(t_{2}-t_{1}\right) \\
L & =c \cdot\left(T_{\text {obs }}-T_{\text {source }}\right)
\end{aligned}
$$

And with the Equation (1.5)

$$
\begin{gathered}
L=c \cdot((1+z)-1) \cdot T_{\text {source }} \\
L=c \cdot z \cdot T_{\text {source }}
\end{gathered}
$$

2) Blueshift Case

See specific configuration of Figure 7.

- The distance of emission source of the photon (B) at the time $t_{1}=T_{\text {source }}$ :

$$
\begin{gathered}
d_{\text {source }}=\lambda \\
\lambda=v_{\text {photon }} T_{\text {source }} \\
\lambda=c T_{\text {source }}
\end{gathered}
$$




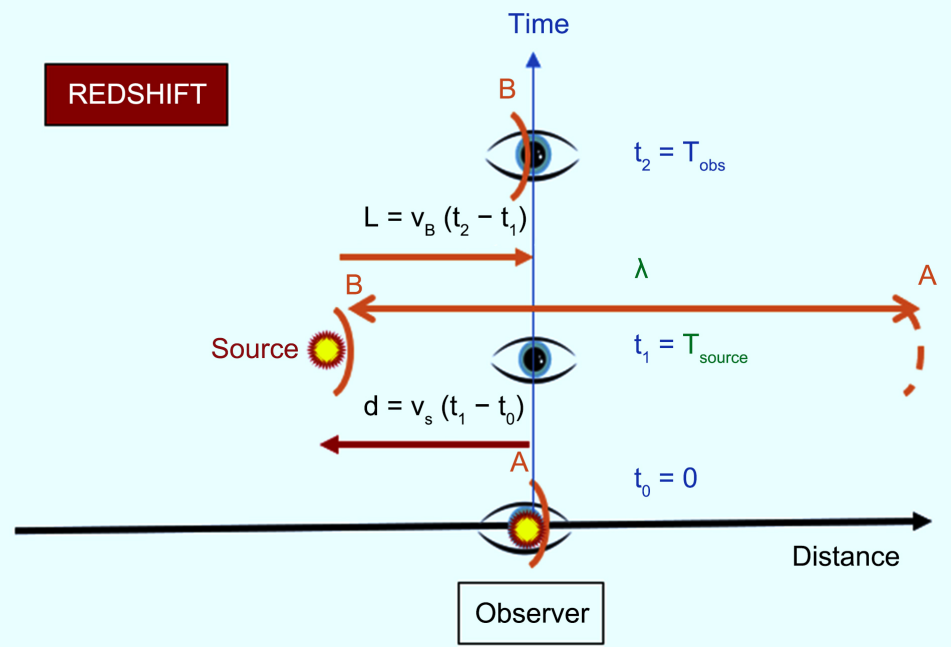

Figure 6. Redshift case/SR.

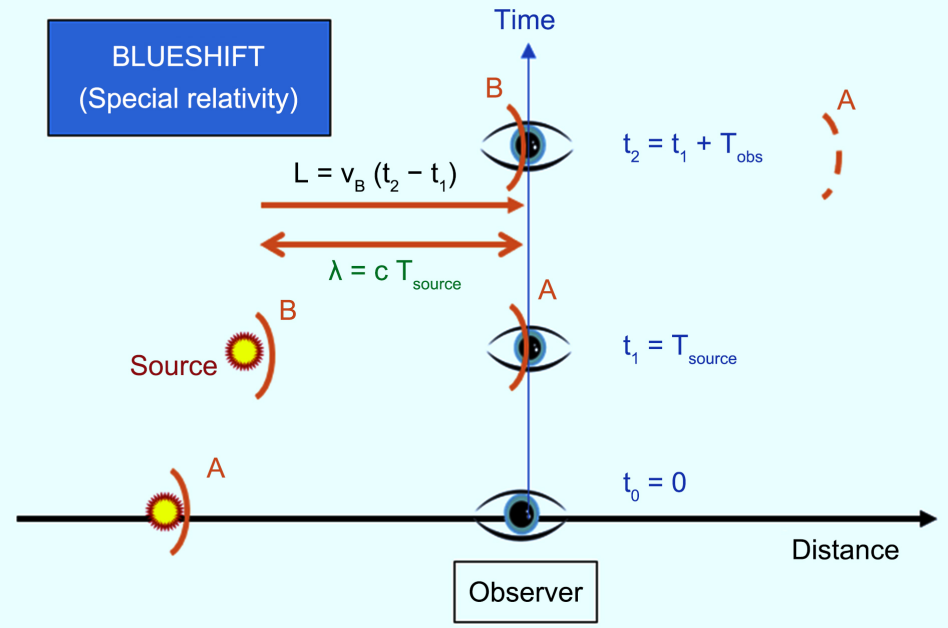

Figure 7. Blueshift case/Special Relativity.

Then

$d_{\text {source }}=c T_{\text {source }}$

- And the length traveled by the photon (B) to reach the observer is 16

$$
\begin{gathered}
L=v_{\text {photon }}\left(t_{2}-t_{1}\right) \\
L=c \cdot T_{\text {obs }} \\
L=c \cdot(1+z) \cdot T_{\text {source }}
\end{gathered}
$$

Numerical application (with for example $T_{\text {source }}=1$ second):

Let us apply in Table 2 previous formulas with some example of $z$ and with the difference of length:

$$
\delta=L-|d|
$$


Table 2. Relativistic numerical application.

\begin{tabular}{ccccc}
\hline $\mathrm{T}=\mathrm{T}$ source $=$ 1 second for example & \multicolumn{2}{c}{ Blueshift } & \multicolumn{2}{c}{ Redshift } \\
\hline shift z & -0.5 & -0.1 & 1 & 10 \\
\hline $\begin{array}{c}\text { Distance of B source from A: } \\
\beta \text { source (SR) }\end{array}$ & -0.60 & -0.10 & 0.60 & 0.98 \\
d: distance of emission of B from O & $\mathrm{c} \mathrm{T}$ & $\mathrm{c} \mathrm{T}$ & $-0.6 \mathrm{c} \mathrm{T}$ & $-0.98 \mathrm{c} \mathrm{T}$ \\
Length traveled by photon B: & & & & \\
T observed/T source $=(1+\mathrm{z})$ & 0.5 & 0.9 & 2 & 11 \\
L: Length traveled by photon B & $0.5 \mathrm{c} \mathrm{T}$ & $0.9 \mathrm{c} \mathrm{T}$ & $\mathrm{c} \mathrm{T}$ & $10 \mathrm{c} \mathrm{T}$ \\
Difference $\delta$ & $-0.5 \mathrm{c} \mathrm{T}$ & $-0.1 \mathrm{c} \mathrm{T}$ & $0.4 \mathrm{c} \mathrm{T}$ & $9.02 \mathrm{c} \mathrm{T}$ \\
\hline
\end{tabular}

So to explain the redshift, you need to suppose a Universe expansion (for example a Universe expansion of $0.4^{*} c^{\star} T$ for $z=1$ ).

And to explain the blueshift, you need to suppose a Universe contraction (of $-0.1^{\star} c^{\star} T$ for $\left.z=-0.1\right)$ !

$\rightarrow$ Without a Universe which would expand by itself, the relativistic formula of redshift is uncoherent! 


\section{Appendix 2. Derivation of the Neo-Newtonian Formula of Redshift}

Let us have a coordinate system oriented according to the propagation of the photons. According to the motion of the Source and the motion of the Observer, there are 4 cases. We illustrate 2 cases (see Figure 8 and Figure 9).

\section{A2.1. The Hypotheses}

1) About the source:

Let us have a source emitting photon (A) at time $t_{0}$ and photon (B) at time $t_{1}$ By definition, the source period $T_{\text {source }}$ is

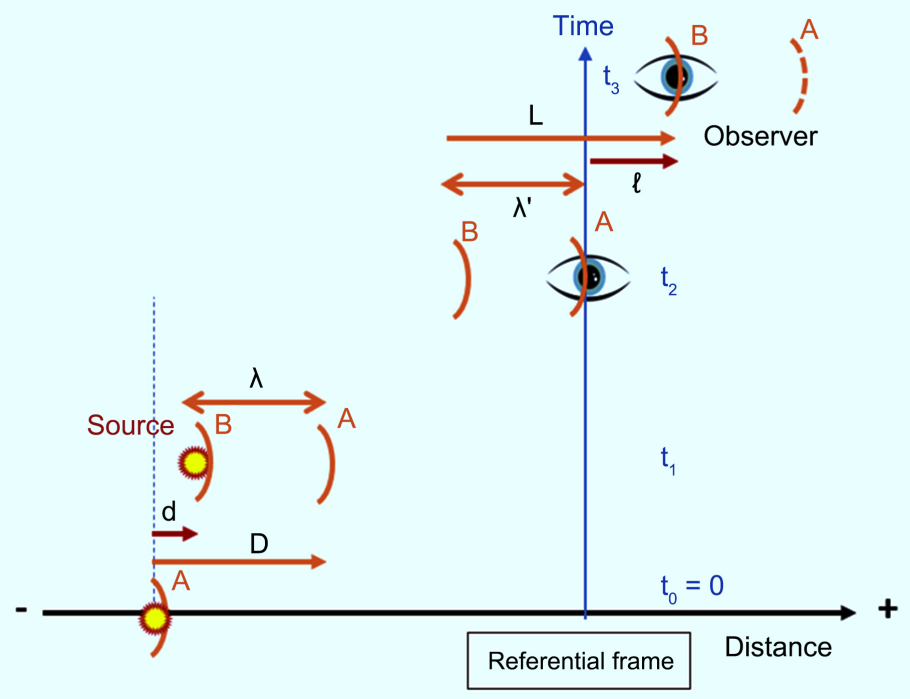

Figure 8. Source motion to the right \& Observer motion to the right.

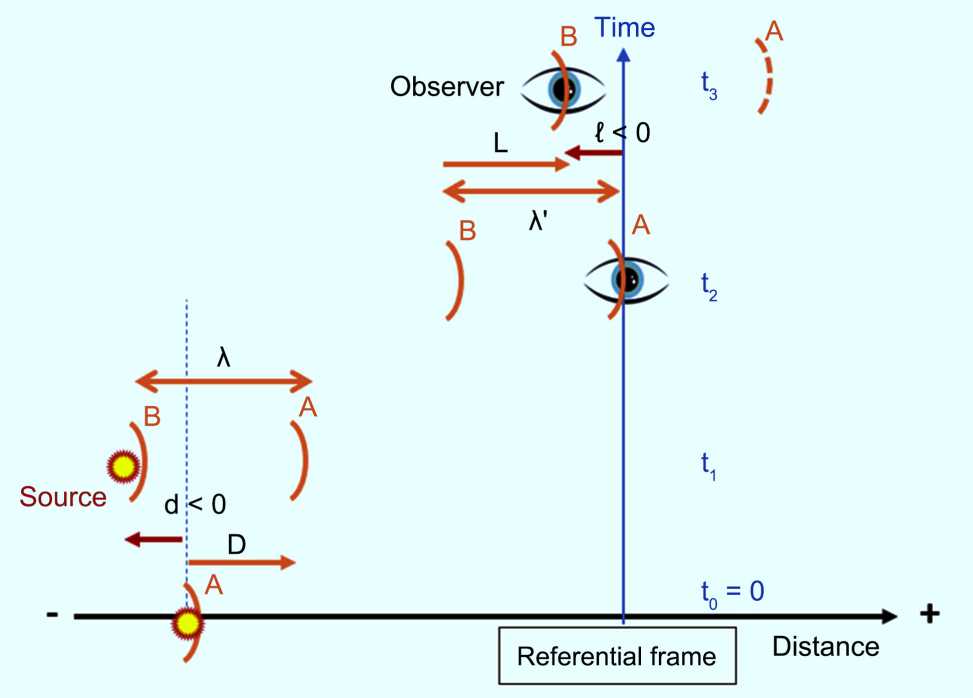

Figure 9. Source motion to the left \& Observer motion to the left. 


$$
T_{\text {source }}=t_{1}-t_{0}
$$

$d_{\text {source }}$ is the distance traveled by the source at the velocity $v_{\text {source }}$ during a period $T_{\text {source }}$

$$
d_{\text {source }}=v_{\text {source }} \cdot T_{\text {source }}
$$

$D_{A}$ is the distance traveled by the photon (A) at the velocity $V_{A}$ during a period $T_{\text {source }}$

$$
D_{A}=v_{A} \cdot T_{\text {source }}
$$

$\lambda$ is the distance between photon (A) and photon (B)

$$
\lambda=D_{A}-d_{\text {source }}
$$

Please note these are algebraic value. If $d_{\text {source }}$ is positive, $\lambda$ will be lower than $D_{A}$. If $d_{\text {source }}$ is negative, $\lambda$ will be greater than $D_{A}$ (please check Figure 8 with $t_{1}$ for $d_{\text {source }}$ positive, and Figure 9 with $t_{1}^{\prime}$ with $d_{\text {source }}$ negative).

\section{2) About the observer:}

Let us have an observer receiving photon (A) at time $t_{2}$ and photon (B) at time $t_{3}$

By definition, the observer period $T_{o b s}$ is

$$
T_{o b s}=t_{3}-t_{2}
$$

$l_{o b s}$ is the distance traveled by the observer at the velocity $v_{o b s}$ during a period $T_{o b s}$

$$
l_{o b s}=v_{o b s} \cdot T_{o b s}
$$

$L_{B}$ is the distance traveled by the photon (B) at the velocity $v_{B}$ during a period $T_{o b s}$

$$
L_{B}=v_{B} \cdot T_{o b s}
$$

$\lambda^{\prime}$ is the distance between photon (A) and photon (B)

$$
\lambda^{\prime}=L_{B}-l_{\text {obs }}
$$

Please note these are algebraic value. If $l_{\text {obs }}$ is positive, $\lambda^{\prime}$ will be lower than $L_{B}$. If $l_{o b s}$ is negative, $\lambda^{\prime}$ will be greater than $L_{B}$ (please check Figure 8 at $t_{3}$ for $l_{o b s}$ positive, and Figure 9 at $t_{3}^{\prime}$ for $l_{o b s}$ negative).

Because the motion of the source is continuous, the velocities of the photons are all the same

$$
v_{B}=v_{A}
$$

Because the velocities of the photons are the same, the distance between photons remain the same

$$
\lambda^{\prime}=\lambda
$$

What is perceived is the frequency, not the wave length. So the redshift $z$ is, with $f$ for frequency

$$
1+z=\frac{f_{\text {source }}}{f_{\text {obs }}}
$$

Remark 1: In Newtonian mechanics and so in Neo Newtonian Mechanics, 
there is no difference of length whatever it is measured from the Observer frame or from the Referential frame.

Remark 2: when $c$ is constant please note Equation (2.11) is equivalent to

$$
1+z=\frac{\lambda_{\text {obs }}}{\lambda_{\text {source }}}
$$

By definition of the frequency

$$
f=\frac{1}{T}
$$

And let us define $V$ the distance speed of the source from the observer by

$$
V \cong v_{\text {source }}-v_{\text {obs }}
$$

In Neo-Newtonian Mechanics, the velocities cannot be directly added for very high velocities. In the present case, we consider that the source and the observer are in usual velocity, so we can use the approximatively equal symbol.

\section{A2.2. Derivation}

Using Equations (2.4), (2.3), (2.2)

$$
T_{\text {source }}=\frac{\lambda}{v_{A}-v_{\text {source }}}
$$

Using equations (2.6), (2.7), (2.8), (2.9), (2.10)

$$
T_{o b s}=\frac{\lambda^{\prime}}{v_{B}-v_{o b s}}
$$

Using equations (2.9) and (2.10)

$$
T_{o b s}=\frac{\lambda}{v_{A}-v_{o b s}}
$$

Using Equations (2.11) and (2.13)

$$
1+z=\frac{T_{\text {obs }}}{T_{\text {source }}}
$$

Then using Equations (2.15) and (2.17)

$$
\begin{gathered}
1+z=\frac{v_{A}-v_{\text {source }}}{v_{A}-v_{\text {obs }}} \\
v_{A}-v_{o b s}+z\left(v_{A}-v_{o b s}\right)=v_{A}-v_{\text {source }} \\
z\left(v_{A}-v_{o b s}\right)=v_{o b s}-v_{\text {source }}
\end{gathered}
$$

So using Equation (2.14)

$$
V \cong-z\left(v_{A}-v_{o b s}\right)
$$

- When $V$ is positive, the source gets closer to the observer, it is the blueshift.

- When $V$ is negative, the source gets away from the observer, it is the redshift.

Lest us now consider 2 cases, either the photon is moving in the same direction than the source $\left(v_{\text {source }}>0\right)$, either the photon is moving in the opposite 
direction from the source $\left(v_{\text {source }}<0\right)$.

In Neo-Newtonian Mechanics, a photon cannot go faster than the asymptotic limit $s$, which means that the faster velocity of the photon is $c$.

$$
\left\{\begin{array}{l}
\text { if }\left[v_{\text {source }}>0\right] \text { then }\left[v_{A}=c\right] \\
\text { if }\left[v_{\text {source }}<0\right] \text { then }\left[v_{A} \cong c+v_{\text {source }}\right]
\end{array}\right.
$$

Please note that in the second case, $v_{A}$ is not greater but lower than $c$, because $v_{\text {source }}<0$.

1) $v_{\text {source }}>0$ (see Figure 10)

Using Equations (2.22) and (2.23 a)

$$
V \cong-z\left(c-v_{o b s}\right)
$$

Please note that usually $v_{o b s} \ll c$, then we get

$$
V \cong-z \cdot c
$$

That means that when the source is coming toward us relatively to the absolute frame, we will get a blueshift except if the Earth is moving away faster the the source.

Physically, this is not the usual case.

2) $v_{\text {source }}<0 \quad$ (see Figure 11)

Using Equations (2.22) and (2.23b)

$$
V \cong-z\left(c+v_{\text {source }}-v_{\text {obs }}\right)
$$

Then with Equation (2.14)

$$
\begin{aligned}
& V \cong-z(c+V) \\
& (1+z) V \cong-z \cdot c
\end{aligned}
$$

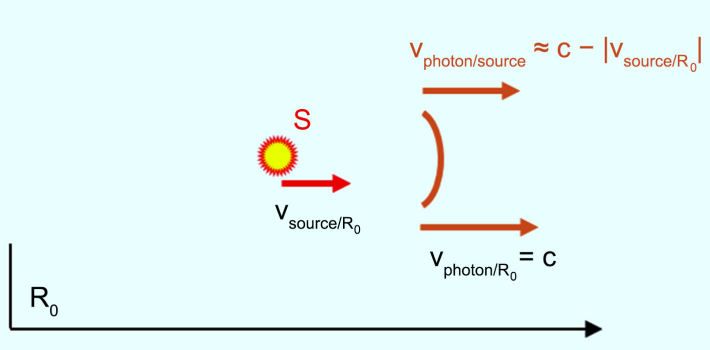

Figure 10. Photon going in the same direction as the source S.

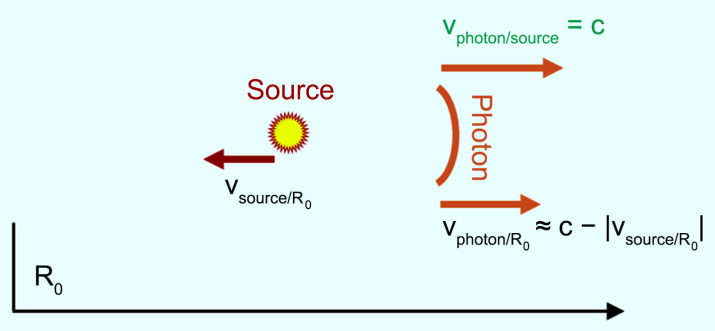

Figure 11. Photon going in the opposite direction of the source S. 
So we get the general formula of the redshift with the algebraic value $V$ :

$$
V \cong-\frac{z}{1+z} c
$$

- When $z$ is positive, $V$ is negative, the source moves away from the observer, it is the redshift.

- When $z$ is negative, $V$ is positive, the source moves toward the observer, it is the blueshift.

Please note that when $z \ll 1$, then we get

$$
V \cong-z \cdot c
$$




\section{Appendix 3. Derivation of the Neo-Newtonian Redshift} Formula

Let us have

- An observer without motion (he is in his own frame)

- A source moving at the velocity $v_{\text {source }}$ (in the observer's frame). So

$$
\beta=\frac{v_{\text {source }}}{c}
$$

NNM equation hereafter for the redshift, when the axis is oriented with the photon

$$
1+z=\frac{1}{1+\beta}
$$

Is equivalent to

$$
\beta=-\frac{z}{1+z}
$$

Period from redshift

$$
\begin{aligned}
& 1+z=\frac{f_{\text {source }}}{f_{\text {obs }}} \\
& 1+z=\frac{T_{\text {obs }}}{T_{\text {source }}}
\end{aligned}
$$

We'll check two cases, the redshift and the blueshift one.

\section{1) Redshift case}

See specific configuration of Figure 12.

- The distance of emission source of the photon (B) at the time $t_{1}=T_{\text {source }}$ :

$$
d_{\text {source }}=v_{\text {source }}\left(t_{1}-t_{0}\right)
$$

$$
d_{\text {source }}=\beta \cdot c \cdot T_{\text {source }}
$$

with $v_{\text {source }}<0$ and so $\beta<0$.

\section{REDSHIFT}

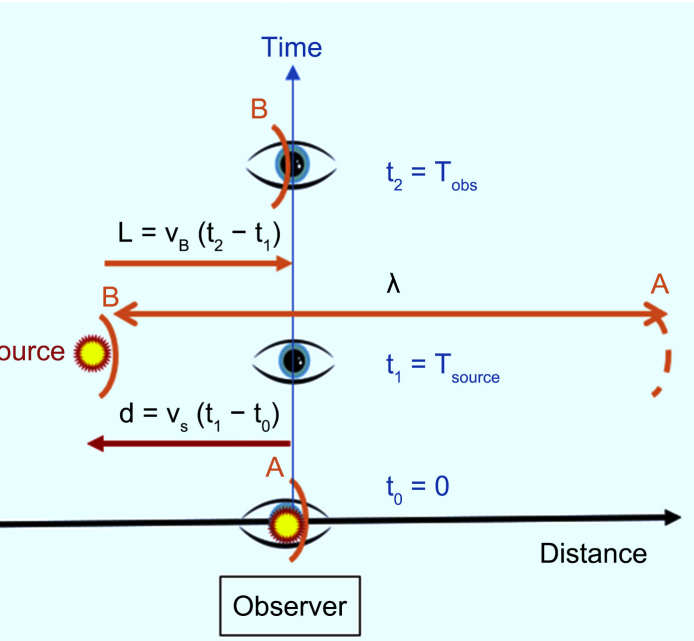

Figure 12. Redshift case/NNM. 
- And the length traveled by the photon (B) to reach the observer is

$$
L=v_{\text {photon }}\left(t_{2}-t_{1}\right)
$$

for the redshift $\left(v_{\text {source }}<0\right)$ using the Equation $(2.23 \mathrm{~b})$ already seen

$$
v_{\text {photon }} \cong c+v_{\text {source }}
$$

with $v_{\text {source }}<0$

$$
L=\left(c+v_{\text {source }}\right) \cdot\left(T_{\text {obs }}-T_{\text {source }}\right)
$$

Using Equations (3.1) and (3.2):

$$
\begin{gathered}
c+v_{\text {source }}=c+\beta \cdot c \\
1+\beta=\frac{1}{1+z}
\end{gathered}
$$

So

$$
c+v_{\text {source }}=\frac{c}{1+z}
$$

And using Equation (3.5)

$$
z \cdot T_{\text {source }}=T_{\text {obs }}-T_{\text {source }}
$$

Then from Equation (3.9)

$$
\begin{gathered}
L=\frac{c}{1+z} \cdot\left(z \cdot T_{\text {source }}\right) \\
L=c \cdot \frac{z}{1+z} \cdot T_{\text {source }}
\end{gathered}
$$

So in the case of the redshift, $\delta$ is the difference

$$
\begin{gathered}
\delta=L-|d| \\
\delta=c \cdot \frac{z}{1+z} \cdot T_{\text {source }}-(|\beta| \cdot c) T_{\text {source }}
\end{gathered}
$$

According to NNM, for the redshift when the axe is oriented with the photon:

$$
|\beta|=\frac{z}{1+z}
$$

Then

$$
\delta=0
$$

\section{2) Blueshift case}

See specific configuration of Figure 13.

- The distance of emission source of the photon (B) at the time $t_{1}=T_{\text {source }}$ :

$$
\begin{gathered}
\lambda=D-d \\
\lambda=v_{\text {photon }} \cdot T_{\text {source }}-v_{\text {source }} \cdot T_{\text {source }}
\end{gathered}
$$




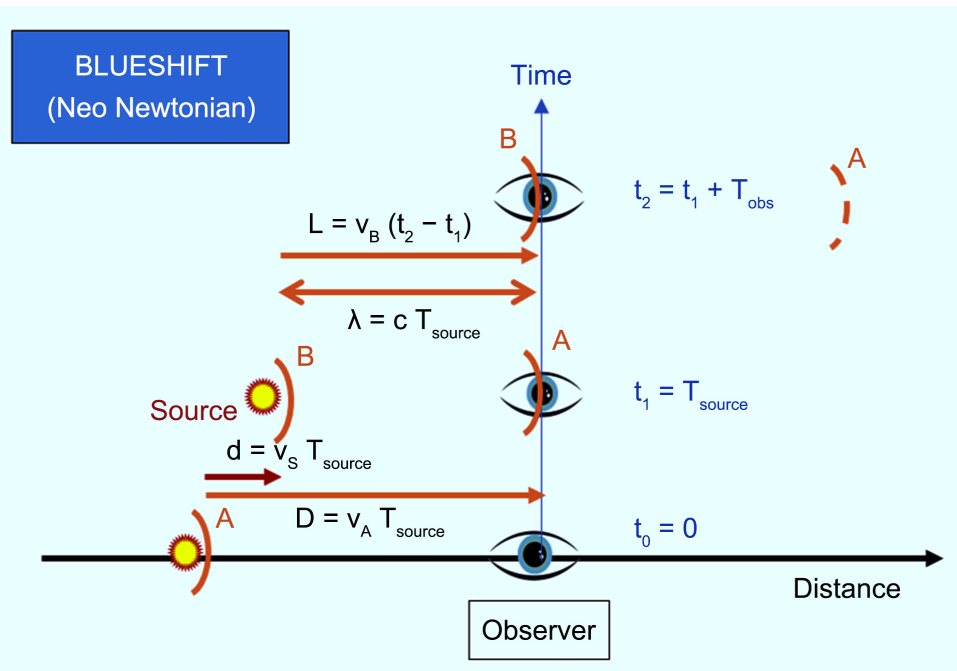

Figure 13. Blue shift case/Neo Newtonian Mechanics.

for the redshift $\left(v_{\text {source }}>0\right)$ using the Equation (2.23a) already seen

$$
\begin{gathered}
v_{\text {photon }}=c \\
\lambda=c \cdot T_{\text {source }}+z \cdot c \cdot T_{\text {source }}
\end{gathered}
$$

with $z<0$

$$
\lambda=c(1+z) \cdot T_{\text {source }}
$$

- And the length traveled by the photon (B) to reach the observer is

$$
\begin{gathered}
L=v_{\text {photon }}\left(t_{2}-t_{1}\right) \\
L=v_{\text {photon }} T_{o b s}
\end{gathered}
$$

Using Equation (3.3)

$$
\begin{aligned}
& T_{\text {obs }}=(1+z) T_{\text {source }} \\
& L=c \cdot(1+z) \cdot T_{\text {source }}
\end{aligned}
$$

So in the case of the blueshift:

$$
\begin{gathered}
\delta=L-\lambda \\
\delta=c \cdot(1+z) T_{\text {source }}-c(1+z) \cdot T_{\text {source }} \\
\delta=0
\end{gathered}
$$

Numerical applications (with $T_{\text {source }}=1$ second).

Let us apply in Chart 3 previous formulas with some example of $z$ and with the difference of length $\delta$ with:

$$
\begin{aligned}
& \text { for the blueshfit, } \delta=L-|\lambda| \\
& \text { for the blueshfit, } \delta=L-|d|
\end{aligned}
$$


Chart 3. Neo-Newtonian numerical application.

\begin{tabular}{ccccc}
\hline T $=$ Tsource = 1 second & \multicolumn{2}{c}{ BLUESHIFT } & \multicolumn{2}{c}{ REDSHIFT } \\
shift $\mathrm{z}$ & -0.5 & -0.1 & 1 & 10 \\
\hline $\begin{array}{c}\text { Distance of B source from A } \\
\beta \text { source (NNM) }\end{array}$ & -1.00 & -0.11 & 0.50 & 0.91 \\
d: distance of emission of B from O & & & $-0.5 \mathrm{c} \mathrm{T}$ & $-0.91 \mathrm{c} \mathrm{T}$ \\
$\lambda$ : distance from B to A & $(0.5 \mathrm{c}) \mathrm{T}$ & $(0.9 \mathrm{c}) \mathrm{T}$ & & \\
Length traveled by photon B: & & & & \\
T observed/Tsource = (1 + z) & 0.5 & 0.9 & 2 & 11 \\
L: Length traveled using Tobs & $\mathrm{c}(0.5 \mathrm{~T})$ & $\mathrm{c}(0.9 \mathrm{~T})$ & $0.5 \mathrm{c} \mathrm{T}$ & $0.91 \mathrm{c} \mathrm{T}$ \\
Difference $\delta$ & 0 & 0 & 0 & 0 \\
\hline
\end{tabular}




\section{Appendix 4. Interferences}

$$
1+z=\frac{f_{\text {source }}}{f_{\text {obs }}}
$$

1) According to NNM for the redshift

$$
\begin{gathered}
\text { if }\left[v_{\text {source }}<0\right] \text { then }\left[v_{A} \cong c-\left|v_{\text {source }}\right|\right] \\
v_{\text {source }}=\frac{z}{1+z} c
\end{gathered}
$$

So

$$
\begin{gathered}
v_{A} \cong c\left(1-\frac{z}{1+z}\right) \\
v_{A} \cong c \frac{1}{1+z} \\
\lambda_{\text {obs }}=\frac{v_{A}}{f_{\text {obs }}} \\
\lambda_{\text {obs }} \cong\left(c \frac{1}{1+z}\right)\left(\frac{1+z}{f_{\text {source }}}\right) \\
\lambda_{\text {obs }} \cong \frac{c}{f_{\text {source }}} \\
\lambda_{\text {obs }} \cong \lambda_{\text {source }}
\end{gathered}
$$

2) And according to NNM for the blueshift

$$
\begin{gathered}
\text { if }\left[v_{\text {source }}>0\right] \text { then }\left[v_{A}=c\right] \\
\lambda_{\text {obs }}=\frac{v_{A}}{f_{\text {obs }}} \\
\lambda_{\text {obs }}=c\left(\frac{1+z}{f_{\text {source }}}\right) \\
\lambda_{\text {obs }} \neq \lambda_{\text {source }}
\end{gathered}
$$

